\title{
The nutritional value of new varieties of high-yielding triticale: Nutrient composition and in vitro digestibility
}

\author{
A.E. Widodo ${ }^{1,2}$, J.V. Nolan ${ }^{1} \&$ P.A. lji $^{1 \#}$ \\ ${ }^{1}$ School of Environmental and Rural Science, University of New England, \\ Armidale, NSW 2351, Australia \\ ${ }^{2}$ Department of Animal Science, University of Papua, Manokwari - West Papua 98314, Indonesia
}

(Received 14 April 2014; Accepted 21 January 2015; First published online 15 March 2015)

\begin{abstract}
Copyright resides with the authors in terms of the Creative Commons Attribution 2.5 South African Licence.
See: http://creativecommons.org/licenses/by/2.5/za

Condition of use: The user may copy, distribute, transmit and adapt the work, but must recognise the authors and the South African Journal of Animal Science.
\end{abstract}

\begin{abstract}
Ten high-yielding cultivars of triticale obtained from a breeding group at the University of New England, harvested in 2008 and 2009, were assessed for nutrient composition and nutrient digestibility. The cultivars tested were AT528, H20, H127, H128, H157, H249, H418, H426, JRCT74 and Tahara. Their nutrient characteristics, including dry matter, crude protein, crude fat, ash and gross energy, starch content and composition, and concentrations of non-starch polysaccharides, minerals, phytate-P content and amino acids, were determined. The in vitro digestibility and viscosity during digestion were also measured. There was low variability between the cultivars tested and harvest years (the difference between wet and dry conditions) had little effect on nutrient composition.
\end{abstract}

Keywords: High-yielding triticale, harvest years, proximate composition, starch, non-starch polysaccharides

\#Corresponding author: piji@une.edu.au

\section{Introduction}

Triticale ( $X$ Triticosecale Wittmack) is the product of a cross between wheat (Triticum) and rye (Secale). It has been developed primarily as a feed grain for livestock owing to its low versatility for the human food market compared with other conventional cereal grains such as wheat (King, 2011). The world production of triticale has more than doubled in recent years, from 6.4 million tonnes in 2006 to 13.7 million tonnes in 2009 (FAO, 2010). Although it is a minor cereal grain compared with wheat, barley, sorghum and maize, the domestic use of this cereal for animal feed increased in Australia, for example from 202200 tonnes in 2006 to 788000 tonnes in 2010 (To et al., 2010).

Since the first commercial triticale cultivars were released in 1969, many studies have been carried out on the development of triticale. Triticale demonstrates many agronomic advantages, including winter hardiness, drought and disease tolerance and excellent productivity potential. However, they exhibit a wide variation in nutrient content between the cultivars (Vieira et al., 1995).

Over the past 25 years, breeding and selection programmes in Australia have been active and are still being undertaken in New South Wales, South Australia and Western Australia to improve the quality and yield of triticale (Andrews et al., 1991). A crop breeding group at the University of New England (UNE) has developed varieties that are higher yielding and more disease resistant and tolerant of acid soil than the current commercial strains, as well as being high in energy content (UNE, 2008). This group has bred cultivars that have broken the 10-tonne-per-hectare barrier for a wheat-related grain crop. In comparison, Australia's average wheat yield is only 2 tonnes/hectare, while in Europe, wheat yields do not exceed 6 to 8 tonnes/hectare (Scanlan, 2005). These varieties hold much promise for livestock industries, which could use this grain as an alternative energy source. However, there have been no studies on their nutritional composition and value as broiler feed ingredients. This study was therefore conducted to evaluate nutrient composition and in vitro digestibility of new triticale cultivars produced by UNE's crop breeding group.

\section{Materials and Methods}

Ten triticale cultivars were investigated. They were obtained from UNE's crop breeding group, harvested from the university's experimental plots at Armidale $\left(30.50^{\circ} \mathrm{S}, 151.65^{\circ} \mathrm{E}\right.$; altitude, $970 \mathrm{~m}$ above sea level) in 2008 and 2009 . According to the breeding group, there were marked differences in harvest 
conditions between the two years, 2009 being wetter $(816.4 \mathrm{~mm})$ than $2008(766.8 \mathrm{~mm})$. The experiment was conducted in the UNE's field laboratory in Armidale. The 10 high-yielding triticale cultivars tested were AT528, H20, H127, H128, H157, H249, H418, H426, JRCT74 and Tahara.

About $5 \mathrm{~kg}$ of each cultivar was received from the breeders and ground to pass through a $1 \mathrm{~mm}$ sieve. Sub-samples were then taken from this bulk for laboratory analyses. For starch granule analysis, a subsample of whole grains was collected and stored in $20 \mathrm{~mL}$ containers. The analyses of the samples are described below.

The dry matter (DM) content was conducted by the following method. Approximately $2 \mathrm{~g}$ were weighed in duplicate into cooled pre-weighed silica crucibles and placed in a forced-air convection oven (Qnaltex Universal Series 2000, Watson Victor Ltd., Perth, Australia), which was preheated to $105^{\circ} \mathrm{C}$. Samples were set at this temperature for $24 \mathrm{~h}$ and then cooled in the desiccator for 40 to $45 \mathrm{~min}$ to a constant weight. The DM was calculated based on the water loss in weight of the sample after drying.

The nitrogen $(\mathrm{N})$ content of the samples was determined according to the Dumas combustion technique (AOAC, 2002; ID 990.03) with a LECO ${ }^{\circledR} \mathrm{FP} 2000$ automatic $\mathrm{N}$ analyser (LECO Corporation, St. Joseph, MI, USA). In order to interpret the detector response as percentage $\mathrm{N}(\mathrm{w} / \mathrm{w})$, calibration was carried out using a primary standard of pure ethylenediaminetetraacetic acid (EDTA). A factor of 5.76 was used to calculate crude protein (CP) from the total N (McKenzie \& Farrell, 1980). Crude fat or ether extract (EE) content was determined on a Soxhlet apparatus (AOAC, 2002; ID 920.39). Ash content was determined according to AOAC (2002; ID 942.05) method, and dry samples were ignited in a Carbolite CWF Furnace 1200 chamber furnace (Carbolite, Sheffield, UK). The gross energy (GE) concentration was determined for individual samples, using IKA ${ }^{\circledR}$ WERKE bomb calorimeter (C 7000, GMBH \& Co., Staufen, Germany). The chamber was calibrated for the heat of combustion of $0.5 \mathrm{~g}$ of benzoic acid $(26.457 \mathrm{~kJ} / \mathrm{g})$ prior to sample analysis. Approximately $0.50 \mathrm{~g}$ of samples was weighed in duplicate and combusted. The GE value was directly obtained digitally as $\mathrm{MJ} / \mathrm{kg}$.

Total starch and resistant starch in the samples were determined with the Megazyme total starch kit (Megazyme International, Ireland), using the enzyme procedure developed by McCleary et al. (1994). The amylose and amylopectin contents were determined with a Megazyme amylose/amylopectin assay kit (Megazyme International Ireland, Bray Business Park, Bray, Ireland) using the selective quantitative precipitation reaction of con-canavalin A (Con A) for amylopectin (Gibson et al., 1997) and by the colorimetric method of iodine binding with amylose (Chrastil, 1987).

Soluble and insoluble non-starch polysaccharides (NSP) of the ground samples were measured as described by Englyst \& Hudson (1993) and Theander \& Westerlund (1993). The free sugars were determined using the supernatant kept for this purpose, by hydrolysing with $1 \mathrm{~mL}$ of $12 \mathrm{M} \mathrm{H}_{2} \mathrm{SO}_{4}$ at $30^{\circ} \mathrm{C}$ for $2 \mathrm{~h}$ (Saeman et al., 1963), then reducing and acetylating the sugar as per the previous two analyses. The released sugars in all three solutions were measured on a gas chromatograph (Hewlett Packard Model 427, Packard Instruments, Sydney, NSW, Australia) after calibration with allose as internal standard.

Quantitative amino acid (AA) analysis of the ground samples of triticale cultivars was conducted at the Australian Proteome Analysis Facility Ltd, Macquarie University. About $100 \mathrm{mg}$ of sample underwent liquid hydrolysis in $6 \mathrm{M} \mathrm{HCl}$ at $110^{\circ} \mathrm{C}$ for $24 \mathrm{~h}$. After hydrolysis, all amino acids except for cysteine and tryptophan were analysed using the Waters AccQTag Ultra Chemistry (Rydalmere NSW, Australia). The samples were analysed in duplicate. Cysteine and tryptophan were not determined in this study because of the method used.

The mineral contents of the ground grain samples were determined by the microwave digestion technique (Yang, et al., 2012). The samples, $0.45 \mathrm{~g}$, were weighed into a Teflon® TFM vessel. In a fume hood, $8 \mathrm{~mL}$ of nitric acid $(70 \% \mathrm{v} / \mathrm{v})$ was added along with $2 \mathrm{~mL}$ of hydrogen peroxide $(30 \% \mathrm{v} / \mathrm{v})$. The solution was swirled to homogenise it. The vessel was closed and introduced to the rotor segment, then tightened, using a torque wrench. The segment was inserted into the microwave cavity and the temperature sensor connected. The microwave programme was run to completion (about $45 \mathrm{~min}$ ) and the rotor cooled by air until the solution reached room temperature. The vessel was opened and the solution quantitatively transferred into a $50-\mathrm{mL}$ volumetric flask. The solution was made to $50 \mathrm{~mL}$ total volume with deionised water and mixed well prior to analysis. The subsequent mineral determination was carried out by radial system Varian model VISTA-MPX Simultaneous, Inductively Coupled Plasma-Optical Emission Spectrometer (Varian ${ }^{\odot}$ Australia Pty Ltd). The phytate-P content of the grain samples was determined using a sensitive method for the rapid determination of phytate-P in cereals and cereal product as described by Haug \& Lantzsch (1983).

In vitro digestibility was carried out according to the method described by Babinszky et al. (1990), with slight modifications. Digestibility was measured for DM, CP and starch. Five hundred $\mathrm{mg}$ of ground samples were digested in triplicate in $12.5 \mathrm{~mL}$ of $0.1 \mathrm{M} \mathrm{HCl}$ containing $4 \mathrm{~g}$ pepsin/L (Sigma Chemical, St. Louis. Mo, USA) and the sample was incubated at $40^{\circ} \mathrm{C}$ for $1.5 \mathrm{~h}$. Then the sample was dissolved in $2 \mathrm{~mL}$ of $110 \mathrm{mg}$ $\mathrm{NaHCO}_{3}$ and $12.5 \mathrm{~mL}$ of potassium phosphate buffer $(\mathrm{pH} 6.8)$ containing $4 \mathrm{~g}$ of pancreatin and $4 \mathrm{~mL}$ of 
amylase per litre. The mixture was incubated at $40^{\circ} \mathrm{C}$ for $3 \mathrm{~h}$. After the incubation period, $2.5 \mathrm{~mL}$ of $\mathrm{NaCO}_{3}$ $(100 \mathrm{~g} / \mathrm{L})$ were added to each tube and the contents were centrifuged at $3500 \times \mathrm{g}$ for $15 \mathrm{~min}$. The supernatant was collected and kept on ice until the viscosity analysis and the residue was repeatedly rinsed with Milli-Q water. The residue then was freeze-dried and used for determination of DM, CP and starch. Starch was calculated by converting from glucose content. The digestibility of DM, CP and starch was calculated by the following equation:

Digestibility $(\%)=$

$\frac{\text { Weight of samples or nutrient }(\mathrm{mg})-\text { Weight of dried residue or residual nutrient }(\mathrm{mg})}{\text { Weight of DM or nutrient in samples }(\mathrm{mg})} \times 100$

The supernatant collected from the in vitro analysis was used to measure viscosity of the grain as described by Bedford \& Classen (1993). The Brookfield DVIII viscometer was used, which was set at $25^{\circ} \mathrm{C}$ with a cP 40 spindle at $100 \mathrm{rpm}$. The values were expressed in centipoise (cP), which is $1 / 100$ dyne second per $\mathrm{cm}^{2}$.

Data were analysed using 2-sample t-test $(\alpha<0.05)$ in Minitab 16 (Minitab, 2010) to determine whether or not the nutrients of the cultivars were different between the two different harvest years. If the $P$-value was less than or equal to the $\alpha$ level, the null hypothesis $\left(\mathrm{H}_{0}\right)$ was rejected and it was concluded that there were significant differences in the nutritional value of the cultivars harvested in 2008 and 2009. The variation in nutrients within the harvest year was evaluated as coefficient of variation (CV). A correlation analysis was conducted to determine the relationship between the parameters.

\section{Results}

The proximate composition of the cultivars in the two harvest years is shown in Table 1. The DM content varied from $865.5(\mathrm{H} 127$; harvested in 2009) to $882.7 \mathrm{~g} / \mathrm{kg}(\mathrm{H} 418 ; 2009)$ and the $\mathrm{CP}$ content ranged between $92.0(\mathrm{H} 128 ; 2009)$ and $127.8 \mathrm{~g} / \mathrm{kg}(\mathrm{H} 418 ; 2008)$. The crude fat content varied from 13.9 (H128; 2008) to $27.4 \mathrm{~g} / \mathrm{kg}$ (Tahara; 2008), while ash concentration was from 16.7 (Tahara; 2008) to $20.0 \mathrm{~g} / \mathrm{kg}$ (H157; 2009) and GE values were between 18.1 (H128; 2009) and $18.5 \mathrm{MJ} / \mathrm{kg}$ (Tahara; 2008). The DM, CP, ash content and GE were more uniform $(P>0.05$; with $\mathrm{CV}<10 \%)$, regardless of harvest year, while EE (CV $>15 \%$ ) was more variable.

The starch content ranged from $577.6(\mathrm{H} 157 ; 2008)$ to $661.6 \mathrm{~g} / \mathrm{kg}(\mathrm{H} 127 ; 2008)$ (Table 2). The values of amylose ranged from 137.9 (Tahara; 2009) to $180.4 \mathrm{~g} / \mathrm{kg}(\mathrm{H} 249 ; 2008)$, whereas the amylopectin content varied from $429.1(\mathrm{H} 157 ; 2008)$ to $499.8 \mathrm{~g} / \mathrm{kg}(\mathrm{H} 128 ; 2009)$. In percentage terms, amylose was $23.2 \%$ $27.8 \%$, while amylopectin varied between $72.2 \%$ and $76.8 \%$. The resistant starch values were between 41.8 (JRCT; 2008) and $50.9 \mathrm{~g} / \mathrm{kg}(\mathrm{H} 128 ; 2009)$. In addition, the amylose : amylopectin ratio ranged from 0.30 (Tahara; 2009) to 0.38 ( $\mathrm{H} 20 ; 2009$ and $\mathrm{H} 249 ; 2008)$. The total starch, amylose, amylopectin, amylose : amylopectin ratio and resistant starch contents were fairly uniform (CV between $3.65 \%$ and $8.47 \%$ ) and were not different $(P>0.05)$ between the harvest years.

The total free sugar values varied from $19.74(\mathrm{H} 157 ; 2009)$ to $32.75 \mathrm{~g} / \mathrm{kg}$ DM $(\mathrm{H} 426 ; 2008)$ (Table 3$)$. Total soluble NSP content ranged between 8.34 (H157; 2009) and $15.09 \mathrm{~g} / \mathrm{kg}$ DM (AT528; 2008) while insoluble NSP was between $79.16(\mathrm{H} 20 ; 2009)$ and $122.06 \mathrm{~g} / \mathrm{kg}$ (Tahara; 2009) (Tables 4 and 5 , respectively). The concentrations of the individual free sugars, soluble and insoluble NSP were uniform ( $P$ $>0.05)$ between the two harvest years. However, the galactose content of free sugars and mannose component of soluble NSP were different $(P<0.05)$ between cultivars harvested in 2008 and 2009.

The AA contents of the triticale cultivars are shown in Table 6 . The cultivars were richest in glutamine and proline. The major essential amino acids in the triticale cultivars were arginine, glycine, valine, leucine and phenylalanine. There were no differences $(P>0.05)$ in amino acid concentrations between the cultivars between the two harvest years as shown by the low CV (from $3.47 \%$ to $12.93 \%$ ).

Considerable variation was observed for the mineral contents of triticale cultivars across the two harvest years as shown in Table 7. Calcium content ranged from $0.31(\mathrm{H} 128 ; 2009, \mathrm{H} 418 ; 2009$ and H426; in 2008) to $0.46 \mathrm{~g} / \mathrm{kg}$ (Tahara; 2008), while the $\mathrm{P}$ content varied from $3.50(\mathrm{H} 418 ; 2009)$ to $4.42 \mathrm{~g} / \mathrm{kg}(\mathrm{H} 157$; 2008). The values for $\mathrm{K}$ content were between 4.0 (H418; 2009) and $6.1 \mathrm{~g} / \mathrm{kg}(\mathrm{H} 157 ; 2009)$. Sulphur content varied from $1.2(\mathrm{H} 128 ; 2009)$ to $1.7 \mathrm{~g} / \mathrm{kg}(\mathrm{H} 249 ; 2009)$ and the $\mathrm{Mg}$ content ranged from $1.4(\mathrm{H} 127 ; 2009)$ to $1.9 \mathrm{~g} / \mathrm{kg}(\mathrm{H} 418 ; 2008)$. The concentrations of $\mathrm{Ca}, \mathrm{K}, \mathrm{S}$ and $\mathrm{Mg}$ were similar $(P>0.05, \mathrm{CV}<30 \%)$ between the two harvest years; however, $P$ content was different $(P=0.07)$ between 2008 and 2009, but it had a low 
Table 1 Proximate composition ( $/ \mathrm{kg} \mathrm{DM}$ ) of 10 triticale cultivars in two harvest years

\begin{tabular}{|c|c|c|c|c|c|c|c|c|c|c|c|c|c|c|c|c|c|c|c|c|c|c|c|}
\hline \multirow{2}{*}{ Item } & \multicolumn{2}{|c|}{ AT528 } & \multicolumn{2}{|c|}{$\mathrm{H} 2 \mathrm{O}$} & \multicolumn{2}{|c|}{ H127 } & \multicolumn{2}{|c|}{$\mathrm{H} 128$} & \multicolumn{2}{|c|}{ H157 } & \multicolumn{2}{|c|}{ H249 } & \multicolumn{2}{|c|}{ H418 } & \multicolumn{2}{|c|}{ H426 } & \multicolumn{2}{|c|}{ JRCT74 } & \multicolumn{2}{|c|}{ Tahara } & \multicolumn{2}{|c|}{$\mathrm{CV}^{1}{ }^{1}$} & \multirow{2}{*}{$\boldsymbol{P}$} \\
\hline & 2008 & 2009 & 2008 & 2009 & 2008 & 2009 & 2008 & 2009 & 2008 & 2009 & 2008 & 2009 & 2008 & 2009 & 2008 & 2009 & 2008 & 2009 & 2008 & 2009 & 2008 & 2009 & \\
\hline Dry matter & 873.4 & 874.9 & 872.6 & 872.2 & 873.4 & 865.5 & 873.1 & 876.3 & 873.3 & 875.6 & 873.6 & 874.9 & 879.1 & 882.7 & 871.2 & 877.9 & 873.2 & 879.1 & 873.1 & 878.0 & 0.24 & 0.52 & 0.210 \\
\hline Crude protein & 115.4 & 106.0 & 120.8 & 124.2 & 115.4 & 124.4 & 124.7 & 92.0 & 125.3 & 115.1 & 118.0 & 125.1 & 127.8 & 114.2 & 121.7 & 118.8 & 122.7 & 127.8 & 116.1 & 108.2 & 3.67 & 9.58 & 0.195 \\
\hline Crude fat & 15.9 & 20.6 & 18.4 & 16.3 & 15.9 & 18.8 & 13.9 & 20.4 & 14.6 & 19.3 & 13.9 & 17.5 & 18.5 & 17.3 & 19.1 & 26.7 & 19.8 & 18.5 & 27.4 & 23.6 & 22.72 & 15.83 & 0.202 \\
\hline Ash & 17.4 & 18.5 & 19.8 & 18.1 & 19.2 & 18.3 & 18.1 & 19.8 & 18.7 & 20.0 & 17.2 & 17.5 & 17.4 & 17.9 & 17.5 & 18.1 & 19.9 & 19.2 & 16.7 & 18.49 & 6.22 & 4.45 & 0.396 \\
\hline Gross energy $^{2}$ & 18.2 & 18.2 & 18.3 & 18.4 & 18.2 & 18.2 & 18.2 & 18.1 & 18.4 & 18.3 & 18.3 & 18.4 & 18.4 & 18.3 & 18.4 & 18.3 & 18.4 & 18.4 & 18.5 & 18.3 & 0.50 & 0.55 & 0.371 \\
\hline
\end{tabular}

${ }^{1} \mathrm{CV}$ : coefficient of variation

${ }^{2} \mathrm{MJ} / \mathrm{kg}$ DM.

Table 2 Total starch, amylose, amylopectin, resistant starch contents ( $/ \mathrm{kg} \mathrm{DM})$ and amylose : amylopectin ratio of 10 triticale cultivars in two harvest years

\begin{tabular}{|c|c|c|c|c|c|c|c|c|c|c|c|c|c|c|c|c|c|c|c|c|c|c|c|}
\hline \multirow{2}{*}{ Item } & \multicolumn{2}{|c|}{ AT528 } & \multicolumn{2}{|c|}{$\mathrm{H} 20$} & \multicolumn{2}{|c|}{$\mathrm{H} 127$} & \multicolumn{2}{|c|}{ H128 } & \multicolumn{2}{|c|}{ H157 } & \multicolumn{2}{|c|}{ H249 } & \multicolumn{2}{|c|}{ H418 } & \multicolumn{2}{|c|}{ H426 } & \multicolumn{2}{|c|}{ JRCT74 } & \multicolumn{2}{|c|}{ Tahara } & \multicolumn{2}{|c|}{$\mathrm{CV}^{1}{ }^{1}$} & \multirow{2}{*}{$P$} \\
\hline & 2008 & 2009 & 2008 & 2009 & 2008 & 2009 & 2008 & 2009 & 2008 & 2009 & 2008 & 2009 & 2008 & 2009 & 2008 & 2009 & 2008 & 2009 & 2008 & 2009 & 2008 & 2009 & \\
\hline Total starch & 653.7 & 634.6 & 622.3 & 612.3 & 661.6 & 653.7 & 638.9 & 657.0 & 577.6 & 613.6 & 652.5 & 606.8 & 592.3 & 612.3 & 626.1 & 590.0 & 604.0 & 612.3 & 592.3 & 595.4 & 4.75 & 3.65 & 0.780 \\
\hline Amylose & 171.2 & 155.5 & 162.1 & 170.2 & 168.4 & 170.1 & 169.7 & 157.3 & 148.5 & 161.2 & 180.4 & 163.1 & 144.8 & 159.8 & 146.5 & 146.6 & 150.1 & 148.1 & 142.9 & 137.9 & 8.47 & 6.59 & 0.786 \\
\hline Amylopectin & 482.5 & 479.1 & 460.2 & 442.1 & 493.2 & 483.5 & 469.2 & 499.8 & 429.1 & 452.3 & 472.1 & 443.7 & 447.5 & 452.5 & 479.6 & 443.4 & 453.9 & 464.2 & 449.4 & 457.5 & 4.16 & 4.25 & 0.833 \\
\hline $\begin{array}{l}\text { Resistant } \\
\text { starch }\end{array}$ & 47.8 & 48.0 & 46.1 & 45.5 & 48.4 & 47.2 & 48.4 & 50.9 & 46.3 & 44.1 & 49.7 & 43.8 & 44.5 & 45.7 & 45.3 & 44.1 & 41.8 & 44.2 & 45.3 & 47.1 & 4.97 & 4.91 & 0.772 \\
\hline Am : Ap & 0.35 & 0.32 & 0.35 & 0.38 & 0.34 & 0.35 & 0.36 & 0.31 & 0.35 & 0.36 & 0.38 & 0.37 & 0.32 & 0.35 & 0.31 & 0.33 & 0.33 & 0.32 & 0.32 & 0.30 & 6.66 & 7.75 & 0.916 \\
\hline
\end{tabular}

${ }^{1} \mathrm{CV}$ : coefficient of variation; $\mathrm{Am}$ : Ap = amylose : amylopectin ratio. 
Table 3 Available free sugar contents ( $\mathrm{g} / \mathrm{kg} \mathrm{DM}$ ) of 10 triticale cultivars in two harvest years

\begin{tabular}{|c|c|c|c|c|c|c|c|c|c|c|c|c|c|c|c|c|c|c|c|c|c|c|c|}
\hline \multirow{2}{*}{ Item } & \multicolumn{2}{|c|}{ AT528 } & \multicolumn{2}{|c|}{ H2O } & \multicolumn{2}{|c|}{ H127 } & \multicolumn{2}{|c|}{ H128 } & \multicolumn{2}{|c|}{ H157 } & \multicolumn{2}{|c|}{ H249 } & \multicolumn{2}{|c|}{ H418 } & \multicolumn{2}{|c|}{ H426 } & \multicolumn{2}{|c|}{ JRCT74 } & \multicolumn{2}{|c|}{ Tahara } & \multicolumn{2}{|c|}{ CV\% ${ }^{1}$} & \multirow{2}{*}{$P$} \\
\hline & 2008 & 2009 & 2008 & 2009 & 2008 & 2009 & 2008 & 2009 & 2008 & 2009 & 2008 & 2009 & 2008 & 2009 & 2008 & 2009 & 2008 & 2009 & 2008 & 2009 & 2008 & 2009 & \\
\hline Arabinose & 0.57 & 0.47 & 0.38 & 0.43 & 0.58 & 0.49 & 0.43 & 0.61 & 0.37 & 0.38 & 0.43 & 0.36 & 0.58 & 0.45 & 0.44 & 0.51 & 0.46 & 0.55 & 0.42 & 0.53 & 17.47 & 15.86 & 0.759 \\
\hline Xylose & 0.21 & 0.00 & 0.15 & 0.19 & 0.28 & 0.21 & 0.22 & 0.25 & 0.17 & 0.00 & 0.19 & 0.15 & 0.29 & 0.00 & 0.21 & 0.19 & 0.00 & 0.00 & 0.00 & 0.19 & 58.34 & 88.59 & 0.256 \\
\hline Mannose & 4.3 & 4.1 & 5.7 & 4.8 & 3.7 & 3.0 & 4.2 & 3.3 & 3.6 & 3.3 & 4.0 & 4.1 & 4.9 & 2.7 & 4.7 & 5.5 & 4.1 & 5.3 & 3.4 & 4.8 & 15.66 & 24.24 & 0.639 \\
\hline Galactose & 1.3 & 2.0 & 1.7 & 2.3 & 1.5 & 1.7 & 1.3 & 1.9 & 1.3 & 1.9 & 1.5 & 2.6 & 1.7 & 1.8 & 1.6 & 2.3 & 1.2 & 1.7 & 1.9 & 2.3 & 15.07 & 14.95 & 0.000 \\
\hline Glucose & 16.6 & 17.8 & 18.5 & 23.2 & 21.2 & 15.1 & 23.1 & 15.8 & 17.9 & 14.2 & 14.3 & 20.0 & 22.9 & 14.9 & 25.6 & 22.2 & 17.9 & 20.6 & 16.5 & 18.6 & 18.39 & 17.59 & 0.444 \\
\hline Total & 23.1 & 24.4 & 26.5 & 31.0 & 27.2 & 20.4 & 29.4 & 21.7 & 23.4 & 19.7 & 20.5 & 27.2 & 30.9 & 19.9 & 32.8 & 30.6 & 23.6 & 28.1 & 22.2 & 26.4 & 15.65 & 17.59 & 0.591 \\
\hline
\end{tabular}

Table 4 Soluble non-starch polysaccharides contents ( $\mathrm{g} / \mathrm{kg} \mathrm{DM})$ of 10 triticale cultivars in two harvest years

\begin{tabular}{|c|c|c|c|c|c|c|c|c|c|c|c|c|c|c|c|c|c|c|c|c|c|c|c|}
\hline \multirow{2}{*}{ Item } & \multicolumn{2}{|c|}{ AT528 } & \multicolumn{2}{|c|}{$\mathrm{H} 20$} & \multicolumn{2}{|c|}{ H127 } & \multicolumn{2}{|c|}{ H128 } & \multicolumn{2}{|c|}{ H157 } & \multicolumn{2}{|c|}{ H249 } & \multicolumn{2}{|c|}{ H418 } & \multicolumn{2}{|c|}{ H426 } & \multicolumn{2}{|c|}{ JRCT74 } & \multicolumn{2}{|c|}{ Tahara } & \multicolumn{2}{|c|}{$\mathrm{CV}^{1}{ }^{1}$} & \multirow{2}{*}{$P$} \\
\hline & 2008 & 2009 & 2008 & 2009 & 2008 & 2009 & 2008 & 2009 & 2008 & 2009 & 2008 & 2009 & 2008 & 2009 & 2008 & 2009 & 2008 & 2009 & 2008 & 2009 & 2008 & 2009 & \\
\hline Arabinose & 5.2 & 5.4 & 4.3 & 3.4 & 4.4 & 3.9 & 3.6 & 4.1 & 3.2 & 2.9 & 3.8 & 3.0 & 3.0 & 3.3 & 2.9 & 3.4 & 3.9 & 3.8 & 5.1 & 4.1 & 20.78 & 19.42 & 0.570 \\
\hline Xylose & 6.9 & 6.9 & 5.7 & 4.5 & 5.8 & 5.0 & 5.1 & 5.4 & 4.3 & 4.0 & 4.7 & 3.7 & 4.1 & 4.5 & 3.6 & 4.4 & 4.7 & 4.9 & 6.3 & 5.1 & 20.31 & 18.73 & 0.500 \\
\hline Mannose & 1.2 & 0.5 & 1.1 & 1.2 & 1.1 & 0.4 & 0.9 & 0.5 & 1.0 & 0.4 & 1.1 & 1.1 & 1.2 & 0.4 & 1.3 & 0.4 & 0.4 & 0.4 & 0.4 & 0.5 & 32.98 & 52.55 & 0.013 \\
\hline Galactose & 1.3 & 1.2 & 1.3 & 1.2 & 1.1 & 1.1 & 0.9 & 1.1 & 1.2 & 1.2 & 1.2 & 1.3 & 1.0 & 1.1 & 1.2 & 1.2 & 1.1 & 1.0 & 1.3 & 1.2 & 11.64 & 7.27 & 1.000 \\
\hline Total & 15.1 & 14.0 & 13.3 & 10.9 & 12.3 & 10.7 & 10.5 & 11.5 & 9.7 & 8.3 & 11.2 & 9.2 & 9.8 & 10.1 & 10.0 & 10.0 & 10.3 & 10.5 & 13.5 & 11.5 & 16.22 & 14.35 & 0.256 \\
\hline
\end{tabular}

${ }^{1} \mathrm{CV}$ : coefficient of variation. 
Table 5 Insoluble non-starch polysaccharides contents (g/kg DM) of 10 triticale cultivars in two harvest years

\begin{tabular}{|c|c|c|c|c|c|c|c|c|c|c|c|c|c|c|c|c|c|c|c|c|c|c|c|}
\hline \multirow{2}{*}{ Item } & \multicolumn{2}{|c|}{ AT528 } & \multicolumn{2}{|c|}{$\mathrm{H} 2 \mathrm{O}$} & \multicolumn{2}{|c|}{ H127 } & \multicolumn{2}{|c|}{ H128 } & \multicolumn{2}{|c|}{ H157 } & \multicolumn{2}{|c|}{ H249 } & \multicolumn{2}{|c|}{ H418 } & \multicolumn{2}{|c|}{ H426 } & \multicolumn{2}{|c|}{ JRCT74 } & \multicolumn{2}{|c|}{ Tahara } & \multicolumn{2}{|c|}{$\mathrm{CV}^{1}{ }^{1}$} & \multirow{2}{*}{$P$} \\
\hline & 2008 & 2009 & 2008 & 2009 & 2008 & 2009 & 2008 & 2009 & 2008 & 2009 & 2008 & 2009 & 2008 & 2009 & 2008 & 2009 & 2008 & 2009 & 2008 & 2009 & 2008 & 2009 & \\
\hline Arabinose & 30.7 & 31.0 & 28.8 & 23.9 & 27.6 & 30.6 & 29.7 & 30.6 & 34.2 & 41.0 & 30.7 & 27.0 & 29.2 & 38.1 & 27.7 & 34.2 & 35.9 & 37.8 & 38.4 & 43.1 & 11.70 & 18.37 & 0.302 \\
\hline Xylose & 42.3 & 39.1 & 38.2 & 30.9 & 36.6 & 38.4 & 36.6 & 36.9 & 44.7 & 50.0 & 38.2 & 32.6 & 36.0 & 48.3 & 36.6 & 43.7 & 44.0 & 47.2 & 48.5 & 50.1 & 10.90 & 17.09 & 0.567 \\
\hline Mannose & 2.8 & 4.2 & 3.4 & 4.7 & 4.1 & 4.6 & 5.1 & 4.4 & 4.3 & 4.3 & 2.9 & 4.2 & 4.0 & 4.6 & 5.1 & 5.5 & 4.2 & 4.3 & 4.8 & 4.9 & 20.81 & 8.76 & 0.110 \\
\hline Galactose & 2.6 & 2.3 & 2.6 & 2.6 & 2.8 & 2.4 & 3.2 & 2.3 & 3.7 & 2.9 & 2.9 & 2.8 & 3.5 & 3.4 & 3.2 & 2.8 & 3.2 & 2.9 & 3.4 & 3.6 & 12.63 & 15.61 & 0.124 \\
\hline Glucose & 33.6 & 30.4 & 27.5 & 27.4 & 31.7 & 26.4 & 26.3 & 23.9 & 36.6 & 33.4 & 28.5 & 31.5 & 31.4 & 35.1 & 28.9 & 28.6 & 30.4 & 30.6 & 33.9 & 36.0 & 10.37 & 12.73 & 0.738 \\
\hline Total & 99.5 & 95.4 & 89.4 & 79.2 & 91.3 & 91.1 & 89.4 & 87.3 & 109.5 & 117.0 & 91.8 & 87.0 & 92.3 & 114.9 & 90.2 & 102.4 & 104.7 & 109.1 & 114.8 & 122.1 & 9.58 & 14.63 & 0.563 \\
\hline
\end{tabular}

${ }^{1} \mathrm{CV}$ : coefficient of variation. 
Table 6 Amino acid concentrations ( $\mathrm{g} / \mathrm{kg} \mathrm{DM})$ of triticale harvested in different years

\begin{tabular}{|c|c|c|c|c|c|c|c|c|c|c|c|c|c|c|c|c|c|c|c|c|c|c|c|}
\hline \multirow{2}{*}{ Item } & \multicolumn{2}{|c|}{ AT528 } & \multicolumn{2}{|c|}{$\mathrm{H} 20$} & \multicolumn{2}{|c|}{ H127 } & \multicolumn{2}{|c|}{ H128 } & \multicolumn{2}{|c|}{ H157 } & \multicolumn{2}{|r|}{$\mathrm{H} 249$} & \multicolumn{2}{|r|}{ H418 } & \multicolumn{2}{|r|}{ H426 } & \multicolumn{2}{|c|}{ JRCT74 } & \multicolumn{2}{|c|}{ Tahara } & \multicolumn{2}{|c|}{$\mathrm{CV}^{1}{ }^{1}$} & \multirow{2}{*}{$P$} \\
\hline & 2008 & 2009 & 2008 & 2009 & 2008 & 2009 & 2008 & 2009 & 2008 & 2009 & 2008 & 2009 & 2008 & 2009 & 2008 & 2009 & 2008 & 2009 & 2008 & 2009 & 2008 & 2009 & \\
\hline Alanine & 5.7 & 5.0 & 5.6 & 5.8 & 5.8 & 5.7 & 5.1 & 4.5 & 5.6 & 5.1 & 5.5 & 6.0 & 5.6 & 6.0 & 5.3 & 5.6 & 5.6 & 6.0 & 5.4 & 5.2 & 3.70 & 9.22 & 0.821 \\
\hline Arginine & 7.6 & 6.6 & 7.5 & 7.4 & 7.7 & 7.1 & 6.6 & 5.4 & 7.6 & 6.6 & 7.4 & 7.9 & 7.0 & 7.6 & 6.9 & 7.4 & 7.5 & 7.5 & 7.4 & 7.0 & 4.89 & 10.04 & 0.283 \\
\hline Aspartic acid & 8.8 & 7.5 & 8.0 & 8.4 & 9.0 & 8.9 & 7.7 & 6.3 & 8.3 & 7.3 & 8.2 & 8.6 & 7.8 & 8.5 & 7.7 & 8.1 & 8.2 & 8.5 & 8.1 & 7.6 & 5.10 & 9.92 & 0.491 \\
\hline Glutamic acid & 36.6 & 30.8 & 35.5 & 34.9 & 37.0 & 37.8 & 30.3 & 24.7 & 34.7 & 33.1 & 36.4 & 36.9 & 43.4 & 37.8 & 35.0 & 32.9 & 34.4 & 37.5 & 33.2 & 28.9 & 9.39 & 13.10 & 0.242 \\
\hline Glycine & 6.1 & 5.7 & 6.3 & 6.2 & 6.1 & 5.8 & 5.7 & 4.6 & 6.2 & 5.7 & 6.3 & 6.4 & 6.1 & 6.3 & 5.7 & 6.0 & 6.2 & 6.3 & 6.1 & 5.9 & 3.56 & 8.86 & 0.333 \\
\hline Histidine & 3.5 & 2.9 & 3.5 & 3.4 & 3.5 & 3.3 & 2.9 & 2.5 & 3.5 & 3.1 & 3.4 & 3.6 & 3.6 & 3.4 & 3.2 & 3.4 & 3.3 & 3.4 & 3.2 & 3.1 & 6.17 & 10.01 & 0.265 \\
\hline Isoleucine & 4.8 & 4.2 & 4.7 & 4.7 & 4.9 & 4.9 & 4.1 & 3.6 & 4.8 & 4.3 & 4.8 & 5.0 & 5.0 & 4.9 & 4.5 & 4.6 & 4.6 & 4.9 & 4.5 & 4.2 & 5.53 & 9.88 & 0.406 \\
\hline Leucine & 9.3 & 8.1 & 9.0 & 8.9 & 9.4 & 9.3 & 8.1 & 6.9 & 9.0 & 8.4 & 9.2 & 9.5 & 9.8 & 9.2 & 8.7 & 8.7 & 8.9 & 9.2 & 8.8 & 8.0 & 5.32 & 9.12 & 0.191 \\
\hline Lysine & 4.8 & 4.3 & 4.7 & 4.7 & 4.8 & 4.7 & 4.3 & 3.8 & 4.7 & 4.3 & 4.6 & 4.9 & 4.4 & 4.6 & 4.4 & 4.7 & 4.6 & 4.6 & 4.6 & 4.8 & 3.77 & 6.89 & 0.488 \\
\hline Methionine & 1.8 & 1.7 & 2.0 & 2.0 & 1.8 & 1.9 & 1.6 & 1.4 & 2.0 & 1.5 & 1.9 & 2.0 & 1.8 & 1.9 & 1.9 & 1.7 & 2.0 & 1.9 & 1.8 & 1.8 & 5.84 & 2.50 & 0.173 \\
\hline Phenylalanine & 6.6 & 5.6 & 6.3 & 6.0 & 6.6 & 6.5 & 5.6 & 4.7 & 6.4 & 5.7 & 6.4 & 6.5 & 7.1 & 6.3 & 6.1 & 6.0 & 6.1 & 6.2 & 6.1 & 6.6 & 6.32 & 9.49 & 0.060 \\
\hline Proline & 13.3 & 11.5 & 13.4 & 12.7 & 13.4 & 13.1 & 11.3 & 9.3 & 13.1 & 12.2 & 13.7 & 13.7 & 15.5 & 13.6 & 13.1 & 12.1 & 12.9 & 13.5 & 12.0 & 13.3 & 8.28 & 11.93 & 0.118 \\
\hline Serine & 6.5 & 5.7 & 6.6 & 6.3 & 6.6 & 6.4 & 5.7 & 4.8 & 6.5 & 6.0 & 6.6 & 6.7 & 7.1 & 6.7 & 6.2 & 6.2 & 6.5 & 6.7 & 6.5 & 6.5 & 5.45 & 9.42 & 0.185 \\
\hline Threonine & 4.6 & 3.9 & 4.4 & 4.4 & 4.6 & 4.5 & 3.9 & 3.4 & 4.4 & 3.9 & 4.4 & 4.7 & 4.6 & 4.4 & 4.3 & 4.2 & 4.4 & 4.4 & 4.5 & 4.6 & 4.71 & 8.94 & 0.111 \\
\hline Tyrosine & 3.0 & 2.5 & 2.8 & 2.7 & 3.1 & 2.5 & 2.5 & 1.9 & 2.7 & 2.7 & 2.9 & 3.0 & 2.8 & 2.8 & 2.8 & 2.6 & 2.9 & 2.8 & 2.6 & 3.0 & 6.38 & 11.58 & 0.066 \\
\hline Valine & 6.5 & 5.6 & 6.4 & 6.4 & 6.6 & 6.4 & 5.5 & 4.9 & 6.4 & 5.8 & 6.4 & 6.8 & 6.5 & 6.5 & 6.1 & 6.2 & 6.3 & 6.5 & 6.1 & 5.7 & 5.08 & 9.39 & 0.350 \\
\hline
\end{tabular}

${ }^{1} \mathrm{CV}$ : coefficient of variation. 
Table 7 Concentrations of macro and trace minerals of triticale cultivars (DM) harvested in different years

\begin{tabular}{|c|c|c|c|c|c|c|c|c|c|c|c|c|c|c|c|c|c|c|c|c|c|c|c|c|}
\hline \multirow{2}{*}{ Item } & & \multicolumn{2}{|c|}{ AT528 } & \multicolumn{2}{|c|}{$\mathrm{H} 2 \mathrm{O}$} & \multicolumn{2}{|c|}{ H127 } & \multicolumn{2}{|c|}{ H128 } & \multicolumn{2}{|c|}{ H157 } & \multicolumn{2}{|c|}{ H249 } & \multicolumn{2}{|c|}{ H418 } & \multicolumn{2}{|c|}{ H426 } & \multicolumn{2}{|c|}{ JRCT74 } & \multicolumn{2}{|c|}{ Tahara } & \multicolumn{2}{|c|}{$\mathrm{CV}^{1}{ }^{1}$} & \multirow{2}{*}{$P$} \\
\hline & & 2008 & 2009 & 2008 & 2009 & 2008 & 2009 & 2008 & 2009 & 2008 & 2009 & 2008 & 2009 & 2008 & 2009 & 2008 & 2009 & 2008 & 2009 & 2008 & 2009 & 2008 & 2009 & \\
\hline $\mathrm{Ca}$ & \multirow{5}{*}{$\mathrm{g} / \mathrm{kg}$} & 0.4 & 0.3 & 0.4 & 0.4 & 0.4 & 0.3 & 0.4 & 0.3 & 0.4 & 0.4 & 0.4 & 0.4 & 0.4 & 0.3 & 0.3 & 0.3 & 0.4 & 0.4 & 0.5 & 0.4 & 11.79 & 15.06 & 0.039 \\
\hline$P$ & & 4.0 & 3.9 & 4.0 & 3.5 & 4.1 & 3.7 & 4.1 & 3.6 & 4.4 & 4.1 & 3.9 & 3.6 & 4.2 & 3.5 & 4.0 & 3.7 & 4.2 & 4.1 & 3.7 & 3.9 & 4.55 & 5.93 & 0.005 \\
\hline $\mathrm{K}$ & & 5.5 & 5.4 & 6.1 & 5.9 & 5.6 & 4.7 & 5.8 & 5.8 & 5.7 & 6.1 & 5.2 & 5.4 & 5.7 & 4.0 & 5.7 & 5.8 & 5.4 & 5.7 & 5.0 & 5.2 & 5.62 & 11.58 & 0.405 \\
\hline$S$ & & 1.3 & 1.5 & 1.5 & 1.7 & 1.5 & 1.4 & 1.5 & 1.2 & 1.5 & 1.3 & 1.5 & 1.7 & 1.5 & 1.4 & 1.6 & 1.2 & 1.6 & 1.5 & 1.4 & 1.3 & 5.88 & 12.77 & 0.293 \\
\hline $\mathrm{Mg}$ & & 1.7 & 1.7 & 1.8 & 1.8 & 1.7 & 1.4 & 1.8 & 1.4 & 1.8 & 1.7 & 1.6 & 1.7 & 1.9 & 1.6 & 1.7 & 1.7 & 1.8 & 1.9 & 1.7 & 1.7 & 4.86 & 9.50 & 0.136 \\
\hline $\mathrm{Na}$ & \multirow{4}{*}{$\mathrm{mg} / \mathrm{kg}$} & 16.6 & 18.9 & 26.4 & 17.7 & 26.8 & 35.0 & 33.1 & 26.4 & 35.0 & 27.1 & 24.2 & 38.8 & 26.7 & 21.4 & 13.8 & 13.9 & 24.6 & 26.5 & 30.1 & 21.4 & 25.62 & 31.29 & 0.755 \\
\hline $\mathrm{Fe}$ & & 40.3 & 42.3 & 42.3 & 42.5 & 40.8 & 32.0 & 42.9 & 30.5 & 44.4 & 41.8 & 39.9 & 37.6 & 40.9 & 30.0 & 46.3 & 43.3 & 39.7 & 40.7 & 41.1 & 42.8 & 5.10 & 14.18 & 0.083 \\
\hline $\mathrm{Mn}$ & & 59.6 & 53.9 & 53.5 & 53.5 & 60.3 & 38.0 & 44.1 & 38.6 & 59.7 & 53.3 & 47.9 & 49.4 & 46.0 & 42.0 & 45.9 & 42.1 & 51.4 & 45.7 & 47.3 & 52.5 & 12.28 & 13.63 & 0.119 \\
\hline $\mathrm{Zn}$ & & 30.5 & 27.2 & 33.3 & 35.7 & 30.8 & 20.0 & 31.9 & 25.2 & 34.9 & 23.9 & 30.3 & 32.3 & 24.1 & 30.0 & 33.8 & 28.1 & 28.9 & 24.0 & 29.9 & 29.1 & 9.80 & 16.51 & 0.078 \\
\hline
\end{tabular}

Table 8 In vitro dry matter, crude protein and starch digestibility $(\%)$ and viscosity $(\mathrm{CP})^{1}$ of triticale cultivars harvested in different years

\begin{tabular}{|c|c|c|c|c|c|c|c|c|c|c|c|c|c|c|c|c|c|c|c|c|c|c|c|}
\hline \multirow{2}{*}{ Item } & \multicolumn{2}{|c|}{ AT528 } & \multicolumn{2}{|c|}{$\mathrm{H} 2 \mathrm{O}$} & \multicolumn{2}{|c|}{$\mathrm{H} 127$} & \multicolumn{2}{|c|}{ H128 } & \multicolumn{2}{|c|}{ H157 } & \multicolumn{2}{|c|}{ H249 } & \multicolumn{2}{|c|}{ H418 } & \multicolumn{2}{|c|}{ H426 } & \multicolumn{2}{|c|}{ JRCT74 } & \multicolumn{2}{|c|}{ Tahara } & \multicolumn{2}{|c|}{$\mathrm{CV}^{2}{ }^{2}$} & \multirow{2}{*}{$P$} \\
\hline & 2008 & 2009 & 2008 & 2009 & 2008 & 2009 & 2008 & 2009 & 2008 & 2009 & 2008 & 2009 & 2008 & 2009 & 2008 & 2009 & 2008 & 2009 & 2008 & 2009 & 2008 & 2009 & \\
\hline Dry matter & 76.95 & 74.20 & 75.28 & 75.38 & 76.30 & 75.44 & 77.50 & 76.79 & 74.01 & 76.47 & 76.47 & 77.34 & 77.63 & 76.23 & 77.36 & 74.56 & 76.86 & 74.41 & 75.38 & 75.48 & 1.52 & 1.40 & 0.154 \\
\hline Crude protein & 57.12 & 55.85 & 57.54 & 52.17 & 49.38 & 52.22 & 53.11 & 49.98 & 51.81 & 39.73 & 42.15 & 60.72 & 48.73 & 42.70 & 37.87 & 43.86 & 42.67 & 48.92 & 40.79 & 50.46 & 14.44 & 12.65 & 0.609 \\
\hline Starch & 83.83 & 87.80 & 84.11 & 83.97 & 87.18 & 85.41 & 85.09 & 87.51 & 85.41 & 84.59 & 84.61 & 83.59 & 84.75 & 87.43 & 87.81 & 86.81 & 86.54 & 84.01 & 85.84 & 85.24 & 1.54 & 1.89 & 0.861 \\
\hline Viscosity & 1.04 & 1.03 & 1.09 & 1.01 & 1.08 & 1.04 & 1.03 & 1.03 & 1.02 & 1.02 & 1.04 & 1.09 & 1.08 & 1.05 & 1.02 & 1.06 & 1.03 & 1.04 & 1.01 & 1.03 & 2.75 & 2.17 & 0.734 \\
\hline
\end{tabular}

${ }^{1} \mathrm{CP}$ (in centipoises) $=1 \mathrm{mPa}$ s (milliPascal second) $=1 / 100$ dyne-second per square centimetre $\left(\mathrm{dyn} \cdot \mathrm{s} / \mathrm{cm}^{2}\right)$
${ }^{2} \mathrm{CV}$ : coefficient of variation. 
variance between cultivars in each harvest year. For $\mathrm{Na}$, the content ranged from $13.8(\mathrm{H} 426 ; 2008)$ to 38.8 $\mathrm{mg} / \mathrm{kg}$ (H249; 2009), whereas Fe content varied from 30.0 (H418; 2009) to $46.3 \mathrm{mg} / \mathrm{kg}(\mathrm{H} 426 ; 2008)$. The concentration of $\mathrm{Mn}$ was between $38.0(\mathrm{H} 127 ; 2009)$ and $60.3 \mathrm{mg} / \mathrm{kg}(\mathrm{H} 127 ; 2008)$ and $\mathrm{Zn}$ content was from $20.0(\mathrm{H} 127 ; 2008)$ to $35.7 \mathrm{mg} / \mathrm{kg}(\mathrm{H} 20 ; 2008)$. The concentrations of $\mathrm{Na}, \mathrm{Fe}, \mathrm{Mn}$ and $\mathrm{Zn}$ were not different between the two harvest years or between cultivars in each harvest year.

Table 8 shows the in vitro digestibility of DM, ranging from $74.0 \%(\mathrm{H} 157 ; 2008)$ to $77.6 \%(\mathrm{H} 418$; 2008), digestibility of $\mathrm{CP}$ values was between $37.9 \%(\mathrm{H} 426 ; 2008)$ and $60.7 \%$ (H429; 2009) and digestibility of starch varied between $83.6 \%$ (AT528; 2009) to $87.8 \%$ (AT528; 2008). In vitro digestibility of DM, CP and starch was similar $(P>0.05)$ between the two harvest years. The difference in digestibility between the cultivars was also low (CV <15\%). In addition, the viscosity of the digesta ranged from 1.01 to $1.09 \mathrm{cP}$. Viscosity of the same cultivars harvested in 2008 was about the same $(P>0.05)$ as that of samples harvested in 2009 , with a CV less than $3 \%$.

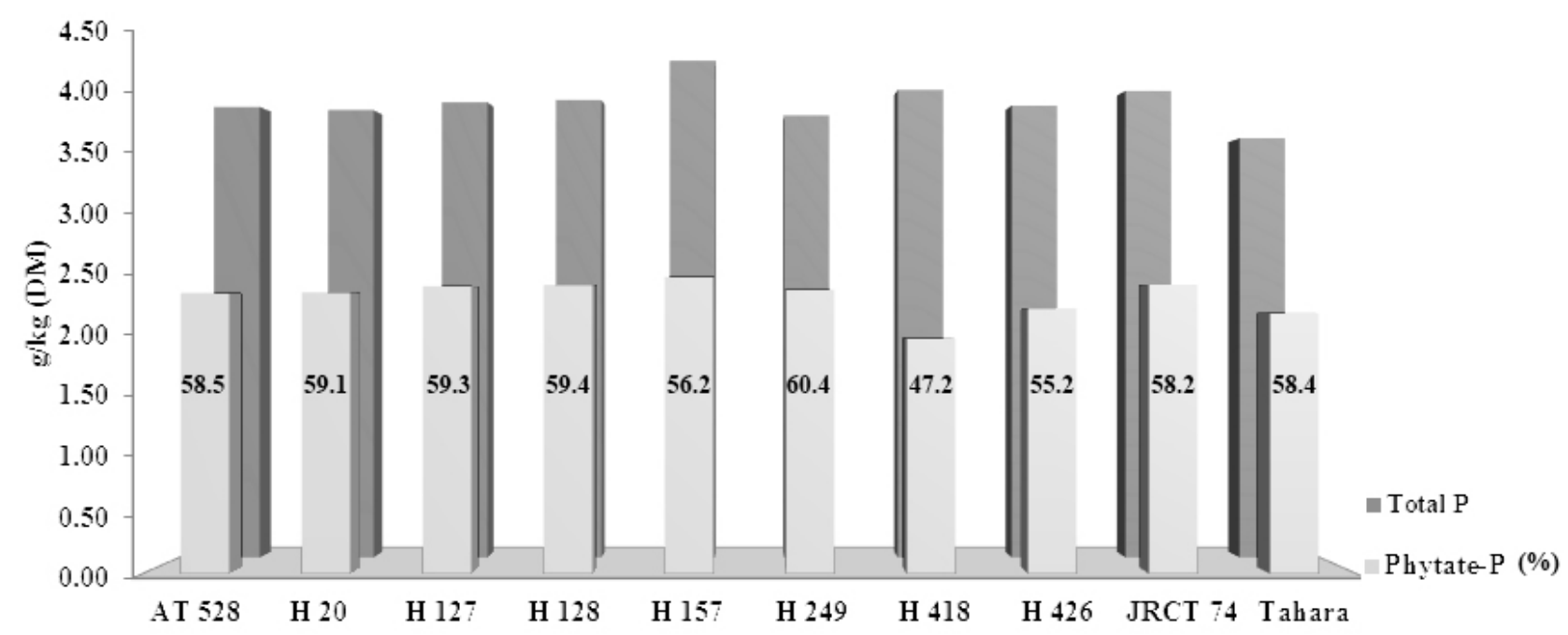

Figure 1 Proportion of phytate-P to total phosphorus $(P)$ in triticale cultivars harvested in 2008.

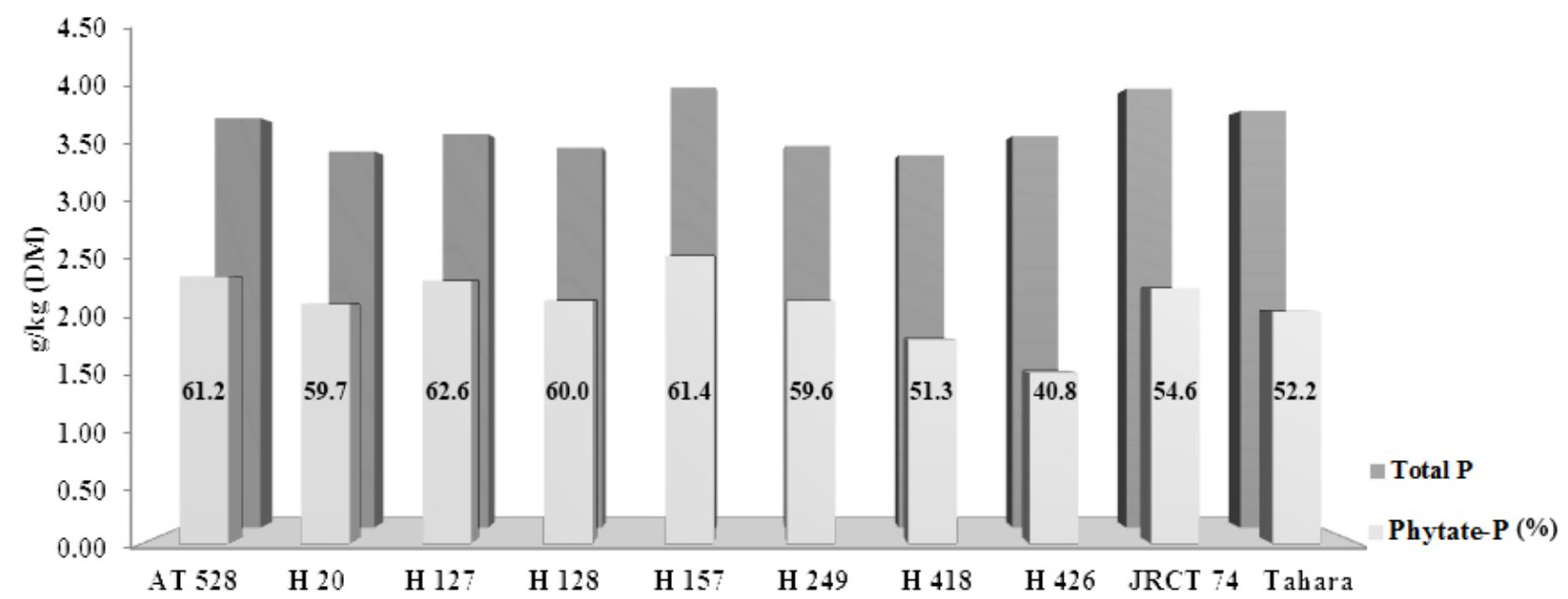

Figure 2 Proportion of phytate-P to total phosphorus (P) in triticale cultivars harvested in 2009.

The phytate-P content of the triticale cultivars harvested in 2008 and 2009 are presented in Figures 1 and 2, respectively. The proportion of phytate-P to total $P$ in the samples harvested in 2008 ranged from $47.2 \%$ to $60.4 \%$, whereas for the samples harvested in 2009 was between $40.8 \%$ and $62.6 \%$. Similar to $P$ 
content, the phytate-P concrentration was different $(P=0.08)$ between the two harvest years, although the variability of phytate-P concentration was relatively low in each year $(6.67 \%$ and $13.88 \%$, in year 2008 and 2009 , respectively). Furthermore, the concentration of phytate-P varied between $1.96(\mathrm{H} 418)$ and $2.48 \mathrm{~g} / \mathrm{kg}$ (H157) in 2008 and $1.80(\mathrm{H} 418)$ and $2.54 \mathrm{~g} / \mathrm{kg}(\mathrm{H} 157)$ in 2009.

A sufficient number of representative granules was not measured to enable detailed statistical analysis, but there appeared to be little difference between the cultivars in starch granular structure. The shape of the starch granules was also similar to that of the parent grains, wheat and rye, which tend to show a bimodal distribution of granule sizes in the mature endosperm of the seed, corresponding with large A- and small B-type starch granules.

\section{Discussion}

The nutrient composition of grains is affected by many factors, including grain variety (genetic factors), growing location (agronomic conditions) and the season or environmental factors (O'Brien, 1999). However, in this study, nutrient composition was similar between the triticale cultivars in two consecutive harvesting years. Such similarities between grain batches have been reported by researchers working at different locations on different cultivars of triticale (Radcliffe et al., 1983 Adeola et al., 1986; Heger \& Eggum, 1991; Salmon et al., 2002; Brand et al., 2003). Triticale appears to have genetic uniformity between the cultivars, although breeding programmes are still keen to improve their quality.

The DM content of triticale in the current study was less than values reported by Radcliffe et al. (1983), Salmon et al. (2002) and Brand et al. (2003), which was about $890 \mathrm{~g} / \mathrm{kg}$ DM or more, but this may be the result of differences in varieties as well as the growth location of the triticale used in the studies. The triticale cultivars used in the current study were the spring varieties, popular in Australia (Cooper et al., 2004) whereas the results reported by Heger \& Eggum (1991) and Brand et al. (2003) were for winter varieties studied in Czechoslovakia and South Africa, respectively, as well as Adeola et al. (1986) and Salmon et al. (2002) in Canada. Other factors that can possibly affect the nutrient composition are weather, management, postharvest storage, pathogens and pests (Morris, 2004).

The protein content of triticale cultivars in this study was about 92 to $128 \mathrm{~g} / \mathrm{kg} \mathrm{DM}$, lower than that reported by Brand et al. (2003) and Salmon et al. (2002), which was 132 to $159 \mathrm{~g} / \mathrm{kg} \mathrm{DM}$, but relatively similar to Radcliffe et al. (1983) and Adeola et al. (1986). In comparison to wheat, McKenzie \& Farrell (1980) and Flores et al. (1994) have reported that triticale has a higher protein value. The crude fat and ash contents of the triticale cultivars in the current study were relatively more variable than those in other studies, which were ranging from 13.9 to $27.4 \mathrm{~g} / \mathrm{kg} \mathrm{DM}$ and 16.7 to $20.0 \mathrm{~g} / \mathrm{kg} \mathrm{DM}$, respectively. With regard to the GE content, there was only a minor variation between the cultivars as well as between the years of harvest. The values are also similar to those obtained in South Africa and Canada (Adeola et al., 1986; Brand et al., 2003), which were ranging from 18.1 to $18.5 \mathrm{MJ} / \mathrm{kg} \mathrm{DM}$.

In previous studies, starch contents of triticale varied from 630 to $730 \mathrm{~g} / \mathrm{kg}$ DM (Leon et al., 1996; Hughes \& Cooper, 2002; Çiftci et al., 2003). The contents found in the current study were lower than these values, the highest starch content being in cultivar $\mathrm{H} 127$, a trial name of triticale cultivar that was later commercialised as 'Bogong'. Although all the triticale samples from the two harvest years contained less total starch than was found in earlier studies, the lower content of resistant starch found in the triticale samples demonstrated that a large proportion of starch fraction may be digestible.

Moran (1982) and Tester et al. (2004) defined a 'normal' amylose content as being between 160 and $350 \mathrm{~g} / \mathrm{kg}$, and the starch is regarded as 'waxy' or 'amylo-' when the amylose content exceeds $350 \mathrm{~g} / \mathrm{kg}$. Based on these definitions, the composition of amylose and amylopectin of the cultivars of H249, AT528, $\mathrm{H} 20, \mathrm{H} 127, \mathrm{H} 128$ and $\mathrm{H} 157$ can be categorised as 'normal' while H418, JRCT74, H426, and Tahara cultivars, which contained less than $160 \mathrm{~g} / \mathrm{kg}$ amylose are 'waxy' cultivars. Cultivar $\mathrm{H} 418$ is a trial name of a cultivar that is now commercialised as 'Canobolas'. In terms of amylose content, all the cultivars are potentially of high digestibility in view of their 'low' amylose contents. Oates (1997) and Carré (2004) postulated that starch with high amylose content $(>40 \%)$ is often considered to be of lower digestibility, i.e. the starch becomes more resistant.

Triticale is more similar to wheat than rye in terms of NSP content. The major fraction of cereal NSP is arabinoxylans (O'Brien, 1999; Dervilly-Pinel et al., 2001). The NSP level has a negative correlation with carbohydrate digestibility (Leeson \& Summers, 2001). Austin et al. (1999) stated that water-insoluble NSP are indigestible by birds whereas soluble NSP are potentially digestible. However, Graham et al. (1993) reported that soluble NSP increase digesta viscosity, which can affect the growth and performance of birds. The new high-yielding triticale cultivars tested in the current study might have less problems of viscosity owing to their low percentage of water-soluble NSP. In addition, the NSP found in the triticale cultivars was mostly insoluble. Data obtained in this study suggest that the concentration of soluble NSP was five times less than that of insoluble NSP. Cultivar AT528 and Tahara had the highest soluble NSP of the cultivars, 
whereas $\mathrm{H} 128$ and $\mathrm{H} 20$ contained the highest level of insoluble NSP. In comparison with NSP content of conventional ingredients used in poultry diets, such as maize and soybean meal, reported by Meng \& Slominski (2005), four of triticale cultivars ( $\mathrm{H} 426, \mathrm{H} 418, \mathrm{H} 157$ and $\mathrm{H} 249$ ) can be classified as low watersoluble NSP cultivars, whereas the other cultivars were slightly higher in NSP content than maize and soybean meal. Based on the total NSP found in the current study, the most common sugars were arabinose and xylose. Digestibility of triticale by birds therefore would likely be beneficial if they were supplemented with microbial enzymes targeting arabinans and xylans, which are the polymers of arabinose and xylose, respectively.

The AA profiles of ingredients are important in formulating poultry diets because supplemental AA are relatively expensive (D'Mello, 2003) and a balanced AA profile in the final diet will have a protein-sparing effect (Brandt et al., 2000). The concentrations of all AA found in the current study were higher than those reported by Sikka et al. (1978), Huet et al. (1988) and Mosse et al. (1988). In addition, the AA concentrations found in these triticale cultivars were also higher than those reported by NRC (1994).

In most cereal grains, lysine is the 'first limiting' AA (Brown, 1989). These results showed that the lysine content of triticale cultivars (3.8 to $4.8 \mathrm{~g} / \mathrm{kg} \mathrm{DM}$ ) was higher than for wheat or rye. This finding is in agreement with the results reported by Stallknecht et al. (1996). Moreover, Heger \& Eggum (1991) and Mosse et al. (1988) reported that lysine levels did not decrease with decreasing protein content and have been reported to be proportionally higher when the protein content of grain was low. Furthermore, the content of lysine in some triticale cultivars was higher in the current study than reported by Flores et al. (1994) and Van Barneveld (2002). In the present study, the highest lysine concentration was in the H249 cultivar, while the lowest was in H128. Elliot (1995) concluded that, after lysine; methionine, threonine, tryptophan, isoleucine and arginine are the next limiting AA in most poultry diets. Tryptophan was not quantified in this study but the concentrations of other potentially limiting AA were high in cultivars H157, $\mathrm{H} 127, \mathrm{H} 418$ and $\mathrm{H} 249$.

With regard to the major elements, $\mathrm{K}$ content was the highest in the triticale cultivars investigated, followed by $\mathrm{P}, \mathrm{Mg}, \mathrm{S}, \mathrm{Ca}$ and $\mathrm{Na}$. The concentration of $\mathrm{Mn}$ was the highest among the trace elements, followed by $\mathrm{Fe}$ and $\mathrm{Zn}$. The similarity in mineral composition between the cultivars as well as between triticale harvested in different years confirms the consistency of the cultivars tested. The difference in $\mathrm{P}$ between 2008 and 2009 may be due to external factors during the growing period, such as water supply, soil type and fertiliser. However, there was low variability in P content between the cultivars. In comparison with previous studies, the range of individual mineral contents corresponded with values reported by Lorenz et al. (1974) and Sehgal et al. (1983). Variations in concentration of mineral elements can be attributed to the effects of soil, water supply, time of sowing, fertilisers, as well as varietal differences. The values in this study indicate that triticale contains relatively higher amounts of the major elements $\mathrm{K}, \mathrm{P}$ and $\mathrm{Na}$ than wheat. The Ca content, on the other hand, is relatively similar to wheat. In the same way, the important minor elements such as $\mathrm{Zn}, \mathrm{Cu}, \mathrm{Fe}$ and $\mathrm{Mn}$ were found to be present in higher amounts in triticale than in wheat as also reported by Lorenz et al. (1974).

The concentrations of phytate-P of triticale cultivars harvested in 2008 and 2009 were relatively similar. The levels of phytate-P were lower than those reported by Singh \& Sedeh (1979) in a study of 14 cultivars of triticale, but they were in the range reported by Singh \& Reddy (1977). In comparison, Kim et al. (2002) reported that the proportion of phytate-P to total $P$ in wheat ranged from $55 \%$ to $79 \%$ in two harvest years. Extra attention should be paid to H157 cultivar when it is included in diets due to its high phytate-P content.

In the present study, in vitro digestibility of DM, starch and CP as well as the digesta viscosity were not different between the cultivars, or between the harvest years. These findings are positive for the rapid selection and use of the cultivars in diet formulation. However, the digestibility of starch is relatively lower than that in the study reported by Flores et al. (1994). The presence of anti-nutritive factors, particularly NSP, may be responsible for the low digestibility of starch and protein. The inclusion of exogenous enzymes such as phytase and/or carbohydrase could help to increase the digestibility of these nutrients. Viscosity values during digestion were relatively similar between the cultivars and this was mainly the result of the similarity in content and types of the NSP.

\section{Conclusion}

It may be concluded from the present study that the nutrient compositions of the 10 cultivars of triticale, harvested in two different years, were generally similar. Phosphorus content was the most variable between the two harvest years. The cultivars were superior, in terms of concentration of key nutrients such as protein and amino acids, to wheat and rye, the cereals from which triticale was bred. 


\section{Acknowledgement} R. Jessop.

The triticale grains were provided by the University New England's crop breeding group, led by

\section{References}

Adeola, O., Young, L.G., McMillan, E.G. \& Moran, E.T., 1986. Comparative protein and energy value of OAC Wintri triticale and corn for pigs. J. Anim. Sci. 63, 1854-1861.

Andrews, A.C., Wright, R., Simpson, P.G., Jessop, R., Reeves, S. \& Wheeler, J., 1991. Evaluation of new cultivars of triticale as dual-purpose forage and grain crops. Aust. J. Exp. Agr. 31, 769-775.

Association of Official Analytical Chemists 2002. Official Methods of Analysis of AOAC International 17th Edition. AOAC International, Maryland, US.

Austin, S.C., Wiseman, J. \& Chesson, A., 1999. Influence of non-starch polysaccharides structure on the metabolisable energy of U.K. wheat fed to poultry. J. Cereal Sci. 29, 77-88.

Babinszky, L., Van Der Meer, J.M., Boer, H. \& Den Hartog, L.A., 1990. An in-vitro method for prediction of the digestible crude protein content in pig feeds. J. Sci. Food Agr. 50, 173-178.

Bedford, M.R. \& Classen, H.L., 1993. An in vitro assay for prediction of broiler intestinal viscosity and growth when fed rye-based diets in the presence of exogenous enzymes. Poult. Sci. 72, 137-143.

Brand, T.S., Cruywagen, C.W., Brandt, D.A., Viljoen, M. \& Burger, W.W., 2003. Variation in the chemical composition, physical characteristics and energy values of cereal grains produced in the Western Cape area of South Africa. S. Afr. J. Anim. Sci. 33, 117-126.

Brandt, D.A., Brand, T.S. \& Cruywagen, C.W., 2000. The use of crude protein content to predict concentrations of lysine and methionine in grain harvested from selected cultivars of wheat, barley and triticale grown in the Western Cape region of South Africa. S. Afr. J. Anim. Sci. 30, 22-25.

Brown, W.L., 1989. Triticale: A Promising Addition to the World's Cereal Grains. Panel on Triticale, National Research Council, Washington, D.C., USA. pp. 116.

Carré, B., 2004. Causes for variation in digestibility of starch among feedstuffs. Wld. Poult. Sci. J. 60, 76-89.

Chrastil, J., 1987. Improved colorimetric determination of amylose in starches or flours. Carbohyd. Res. 159, 154-158.

Çiftci, I., Yenice, E. \& Eleroglu, H., 2003. Use of triticale alone and in combination with wheat or maize: effects of diet type and enzyme supplementation on hen performance, egg quality, organ weights, intestinal viscosity and digestive system characteristics. Anim. Feed. Sci. Tech. 105, 149-161.

Cooper, K.V., Jessop, R.S. \& Darvey, N.L., 2004. Triticale in Australia. In: Triticale Improvement and Production, Eds: Mergoum, M. \& Gomez, M.H., Food and Agriculture Organization of the United Nations, Rome. pp. 87-92.

D'Mello, J.P.F., 2003. Amino Acids in Animal Nutrition. (2nd ed.). CABI Publishing, Wallingford, UK. pp. 519.

Dervilly-Pinel, G., Rimsten, L., Saulnier, L., Andersson, R. \& Åman, P., 2001. Water-extractable arabinoxylan from pearled flours of wheat, barley, rye and triticale. Evidence for the presence of ferulic acid dimers and their involvement in gel formation. J. Cereal. Sci. 34, 207-214.

Elliot, M.A., 1995. Feeding and managing pullets to optimize genetic potential. Paper presented at the California Nutritional Conference, (Proceedings Vol. pp. 149-175), California, US.

Englyst, H.N. \& Hudson, G.J., 1993. Dietary fiber and starch: Classification and measurement. In: CRC Handbook of Dietary Fiber and Human Nutrition. (2nd ed.). Ed: Spiller, G., CRC Press Boca Raton, Florida, US. pp. 53-71.

FAO, 2010. Food and Agriculture Organization of the United Nations. Retrieved from http://faostat.fao.org/site/339/default.aspx

Flores, M.P., Castanon, J.I.R. \& McNab, J.M., 1994. Nutritive-value of triticale fed to cockerels and chicks. Br. Poult. Sci. 35, 527-536.

Gibson, T.S., Solah, V.A. \& McCleary, B.V., 1997. A procedure to measure amylose in cereal starches and flours with concanavalin A. J. Cereal Sci. 25, 111-119.

Graham, H., Bedford, M. \& Choct, M., 1993. High gut viscosity can reduce poultry performance. Feedstuffs and Poultry International 65, 1-4.

Haug, W. \& Lantzsch, H.J., 1983. Sensitive method for the rapid determination of phytate in cereals and cereal products. J. Sci. Food Agr. 34, 1423-1426.

Heger, J. \& Eggum, B.O., 1991. The nutritional values of some high-yielding cultivars of triticale. J. Cereal Sci. 14, 63-71.

Huet, J., Baudet, J., Kaab, L.B.B. \& Mosse, J., 1988. Variation of the amino acid scores and of the nitrogento protein conversion factors in barley grain as a function of nitrogen content as compared with wheat and rye. Plant Foods Hum. Nutr. 38, 175-188. 
Hughes, R.J. \& Cooper, K.V., 2002. Nutritive value of triticale for broiler chickens is affected by variety, weather conditions and growth site. Paper presented at the Australian Poultry Science Symposium, (Proceedings Vol. 14, pp. 131-134), Sydney, New South Wales Australia.

Kim, J.C., Mullan, B.P., Selle, P.H. \& Pluske, J.R., 2002. Levels of total phosphorus, phytate-phosphorus, and phytase activity in three varieties of Western Australian wheats in response to growing region, growing season, and storage. Aust. J. Agric. Res. 53, 1361-1366.

King, R., 2011. Triticale: stock feed guide. Retrieved from www.waratahseeds.com.au/content/Triticaleguide-stockfeed.pdf.

Leeson, S. \& Summers, J.D., 2001. Scott's Nutrition of the Chicken.(4th ed.). Nottingham University Press, Nottingham, England. pp. 601.

Leon, A.E., Rubiolo, A. \& Anon, M.C., 1996. Use of triticale flours in cookies: Quality factors. Cereal Chem. 73, 779-784.

Lorenz, K., Reuter, F.W. \& Sizer, C., 1974. The mineral composition of triticales and triticale milling fractions by $\mathrm{X}$-ray fluorescence and atomic absorption. Cereal Chem. 51, 534-541.

McCleary, B.V., Gibson, T.S., Solah, V. \& Mugford, D.C., 1994. Total starch measurement in cereal products: Interlaboratory evaluation of a rapid enzymic test procedure. Cereal Chem. 71, 501-505.

McKenzie, R.J. \& Farrell, D.J., 1980. How useful is triticale for pigs and poultry? In: Recent Advances in Animal Nutrition in Australia, Ed: Farrell, D.J., University of New England, Armidale, NSW Australia. pp. 91-110.

Meng, X. \& Slominski, B., 2005. Nutritive values of corn, soybean meal, canola meal, and peas for broiler chickens as affected by a multicarbohydrase preparation of cell wall degrading enzymes. Poult. Sci. $84,1242-1251$.

Moran, E.T., 1982. Starch digestion in fowl. Poult. Sci. 61, 1257-1267.

Morris, C.F., 2004. Cereals/Grain - Quality attributes In: Encyclopedia of Grain Science, Eds: Wrigley, C., Corke, H. \& Walker, C.E., Elsevier Science, London, UK. pp. 238-254.

Mosse, J., Huet, J.C. \& Baudet, J., 1988. The amino acid composition of triticale grain as a function of nitrogen content: comparison with wheat and rye. J. Cereal Sci. 7, 49-60.

NRC, 1994. National Research Council; Nutrient Requirements of Poultry: Ninth Revised Edition. The National Academies Press, Washington, US. pp. 176.

O'Brien, L., 1999. Genotype and environment effects on feed grain quality. Aust. J. Agr. Res. 50, 703-719.

Oates, C.G., 1997. Towards an understanding of starch granule structure and hydrolysis. Trends Food Sci Tech. 8, 375-382.

Radcliffe, B.C., Egan, A.R. \& Driscoll, C.J., 1983. Nutritional evaluation of triticale grain as an animal feed. Aust. J. Exp. Agr. 23, 419-425.

Saeman, J.F., Moore, W.E. \& Millet, M.A., 1963. Sugar unit presents. Hydrolysis and quantitative paper chromatography. In: Methods in Carbohydrate Chemistry. Ed: Whristler, R.L., Vol. 03, Academic Press, New York. pp. 54-69.

Salmon, D., Temelli, F. \& Spence, S., 2002. Chemical composition of Western Canadian triticale varieties. Paper presented at the The 5th International Triticale Symposium, 30 June - 5 July, (Proceedings Vol. II, pp. 445-450), Radzikow, Poland.

Scanlan, J., 2005. More than 100 varieties of triticale on display at field day. Retrieved from http://www.une.edu.au/news/archives/000395.html.

Sehgal, K.L., Bajaj, S. \& Sekhon, K.S., 1983. Studies on the composition, quality and processing of triticale; Part II. Mineral composition of grain and flour. Die Nahrung 27, 39-44.

Sikka, K.C., Duggal, S.K., Singh, R., Gupta, D.P. \& Joshi, M.G., 1978. Comparative nutritive value and amino acid content of triticale, wheat, and rye. J. Agric. Food Chem. 26, 788-791.

Singh, B. \& Reddy, N.R., 1977. Phytic acid and mineral compositions of triticales. J. Food Sci. 42, 1077-1083.

Singh, B. \& Sedeh, H.G., 1979. Characteristics of phytase and its relationship to acid phosphates and certain minerals in triticale. Cereal Chem. 56, 267-272.

Stallknecht, G.F., Gilbertson, K.M. \& Ranney, J.E., 1996. Alternative wheat cereals as food grains: Einkorn, emmer, spelt, kamut, and triticale. In: Progress in New Crops: Proceedings of the Third National Symposium, Ed. Janick, J., ASHS Press, Alexandria, US. pp. 156-170.

Tester, R.F., Karkalas, J. \& Qi, X., 2004. Starch structure and digestibility Enzyme-Substrate relationship. Wrld Poult. Sci. J. 60, 186-195.

Theander, O. \& Westerlund, E., 1993. Determination of individual component of dietary fibre. In: CRC Handbook of Dietary Fiber and Human Nutrition. (2nd ed.). Ed: Spiller, G., pp. 77-98. CRC Press Boca Raton, Florida, US. 
To, H., Brown, A., Fell, J., Moloney, J. \& Georgeson, L., 2010. Australian Crop Report. ABARES (Australian Bureau of Agricultural and Resource Economics and Sciences), Canberra.

UNE, 2008. Research Highlight 2008. Retrieved from www.une.edu.au/researchservices/researchhighlights2008.pdf.

Van Barneveld, R.J., 2002. Triticale: A Guide to the use of triticale in livestock feeds. Grains Research Development Corporation, Kingston, Australia. pp. 12.

Vieira, S.L., Penz Jr., A.M., Kessler, A.M. \& Catellan Jr., E.V., 1995. A nutritional evaluation of triticale in broiler diets. J. Appl. Poultry Res. 4, 352-355.

Yang, L., Yan, Q., Cao, Y. \& Zhang, H., 2012. Determination of mineral elements of some coarse grains by microwave digestion with Inductively Coupled Plasma Atomic Emission Spectrometry. E-Journal of Chemistry 9, 93-98. 